\title{
Infinite Subharmonic Solutions of the Forced Relativistic Oscillators*
}

\author{
Zaihong Wang1, Tiantian $\mathrm{Ma}^{2}$ \\ ${ }^{1}$ School of Mathematical Sciences, Capital Normal University, Beijing, China \\ ${ }^{2}$ Editorial Department of Journal, Capital Normal University, Beijing, China \\ Email: zhwang@cnu.edu.cn, matt@cnu.edu.cn
}

How to cite this paper: Wang, Z.H. and Ma, T.T. (2019) Infinite Subharmonic Solutions of the Forced Relativistic Oscillators. Journal of Applied Mathematics and Physics, 7, 1498-1512.

https://doi.org/10.4236/jamp.2019.77101

Received: May 30, 2019

Accepted: July 16, 2019

Published: July 19, 2019

Copyright () 2019 by author(s) and Scientific Research Publishing Inc. This work is licensed under the Creative Commons Attribution-NonCommercial International License (CC BY-NC 4.0). http://creativecommons.org/licenses/by-nc/4.0/

\begin{abstract}
In this paper, we study the multiplicity of subharmonic solutions of the nonlinear differential equation of the forced relativistic oscillators. By using the generalized Poincaré-Birkhoff fixed point theorem, we prove that the equation has infinite subharmonic solutions provided that $g$ satisfies at most linear growth condition.
\end{abstract}

\section{Keywords}

Relativistic Oscillator, Subharmonic Solution, Poincaré-Birkhoff Fixed Point Theorem

\section{Introduction}

We are concerned with the multiplicity of subharmonic solutions of the nonlinear differential equation of the forced relativistic oscillators

$$
\begin{aligned}
& \frac{\mathrm{d}}{\mathrm{d} t}\left(\frac{x^{\prime}}{\sqrt{1-x^{\prime 2}}}\right)+g(x) \\
& =p(t),
\end{aligned}
$$

where $g: \mathbf{R} \rightarrow \mathbf{R}$ is locally Lipschitz continuous, $p: \mathbf{R} \rightarrow \mathbf{R}$ is continuous and periodic, whose least period is $2 \pi$.

The dynamical properties of relativistic oscillators are being studied with an increasing interest because of its extensive applications in different branches of theoretical physics such as quantum mechanics, statistical mechanics, superconductivity theory, nuclear physics and so on (see [1]-[11] and the references therein). In [1], using variational methods, Brezis and Mawhin proved the existence of a $T$-periodic solution of the forced relativistic Pendulum

*Research supported by National Natural Science Foundation of China, No.11501381. 


$$
\frac{\mathrm{d}}{\mathrm{d} t}\left(\frac{x^{\prime}}{\sqrt{1-x^{\prime 2}}}\right)+a \sin x=h(t),
$$

where $a$ is a positive constant and $h$ is a continuous and $T$-periodic function with mean value $\bar{h}=\frac{1}{T} \int_{0}^{T} h(t) \mathrm{d} t=0$. Under same conditions, using Szulkins critical point theory, Bereanu and Torres [2] proved the existence of a second $T$-periodic solution of Equation (1.2) which is not different from the previous by a multiple of $2 \pi$. When the mean value $\bar{h} \neq 0$, using degree arguments, Bereanu and Mawhin [3] proved that Equation (1.2) has at least two solutions not differing by a multiple of $2 \pi$ if

$$
T<\pi \sqrt{3}, \quad|\bar{h}|<a \cos \left(\frac{\pi}{2 \sqrt{3}}\right) .
$$

For the existence of periodic solutions of Equation (1.1) when $g$ is not periodic, it was proved in [4] that Equation (1.1) has at least one $2 \pi$-periodic solution provided that $g$ satisfies

$$
\lim _{|x| \rightarrow \infty} \operatorname{sgn}(x) g(x)=+\infty .
$$

A natural question is whether Equation (1.1) has multiple periodic solutions when $\left(g_{1}\right)$ holds. In the present paper, we shall study this problem. We assume that $g$ still satisfies at most linear condition, i.e. there are two constants $a>0, b>0$ such that

$$
|g(x)| \leq a|x|+b, \text { for every } x \in \mathbf{R} .
$$

By using the generalized Poincaré-Birkhoff fixed point theorem [12], we prove the following theorem.

Theorem 1.1. Assume that conditions $\left(g_{1}\right)$ and $\left(g_{2}\right)$ hold. Then there is an integer $n_{0} \geq 2$ such that, for any integer $n \geq n_{0}$, Equation (1.1) has at least two subharmonic solutions $x_{n, i}(t) \quad(i=1,2)$ of order $n$ and these subharmonic solutions extend to the infinity; that is

$$
\lim _{n \rightarrow \infty}\left(\min _{t \in[0,2 n \pi]}\left(x_{n, i}^{2}(t)+\frac{1}{1-x_{n, i}^{\prime 2}(t)}\right)\right)=+\infty, \quad(i=1,2) .
$$

Throughout this paper, we always use $\mathbf{R}, \mathbf{N}$ to denote the real number set and the natural number set, respectively. For the continuous $2 \pi$-periodic function $p(t)$, we set $\|p\|_{\infty}=\max \{|p(t)|: t \in[0,2 \pi]\}$.

The rest of the paper is organized as follows. Section 2 presents several preliminary lemmas for the equivalent system of Equation (1.1). Section 3 gives some estimates on the angle variable of the transformed system. Section 4 proves the main conclusion (Theorem 1.1).

\section{Basic Lemmas}

Firstly, we consider the equivalent planar system of Equation (1.1). Let us set

$$
y=\frac{x^{\prime}}{\sqrt{1-x^{\prime 2}}} .
$$


Then we have

$$
x^{\prime}=\phi(y)=\frac{y}{\sqrt{1+y^{2}}} .
$$

Obviously, $\phi: \mathbf{R} \rightarrow(-1,1)$ is continuously differentiable. Thus Equation (1.1) is equivalent to the system

$$
\left\{\begin{array}{l}
x^{\prime}=\phi(y), \\
y^{\prime}=-g(x)+p(t) .
\end{array}\right.
$$

For any $\left(x_{0}, y_{0}\right) \in \mathbf{R}^{2}$, we denote by $(x(t), y(t))=\left(x\left(t, x_{0}, y_{0}\right), y\left(t, x_{0}, y_{0}\right)\right)$ the solution of Equation (2.1) satisfying the initial value

$$
x(0)=x_{0}, y(0)=y_{0} .
$$

Next, we shall perform some phase plane analysis for Equation (2.1). Set

$$
G(x)=\int_{0}^{x} g(s) \mathrm{d} s, \Phi(y)=\int_{0}^{y} \phi(s) \mathrm{d} s=\sqrt{1+y^{2}}-1 .
$$

Lemma 2.1. Assume that $\left(g_{1}\right)$ holds. Then every solution $(x(t), y(t))$ of Equation (2.1) exists uniquely on the whole $t$-axis.

Proof. We define a function $W: \mathbf{R}^{2} \rightarrow \mathbf{R}$,

$$
W(x, y)=G(x)+\Phi(y) \text {. }
$$

Obviously, we have

$$
\lim _{|x|+|y| \rightarrow+\infty} W(x, y)=+\infty
$$

Set

$$
W(t)=W(x(t), y(t))=G(x(t))+\Phi(y(t)) .
$$

Then we have

$$
W^{\prime}(t)=\phi(y(t)) p(t) \text {. }
$$

Since $|\phi(y)|<1$, for any $y \in \mathbf{R}$ and $p(t)$ is continuous, we know that

$$
\left|W^{\prime}(t)\right| \leq\|p\|_{\infty}
$$

which implies that, for any positive constant $v>0$,

$$
W(t) \leq W(0)+\|p\|_{\infty} v, \text { for } t \in[0, v) .
$$

Therefore, there is no blow-up for the solution $(x(t), y(t))$ on any finite interval $[0, v)$. Consequently, $(x(t), y(t))$ exists on the whole interval $[0,+\infty)$. Similarly, we can prove that $(x(t), y(t))$ exists on the whole interval $(-\infty, 0]$. The uniqueness of $(x(t), y(t))$ follows directly from the local Lipschitzian condition of $g$ and the differentiability of $\phi$.

We now take the transformation

$$
x(t)=r(t) \cos \theta(t), y(t)=r(t) \sin \theta(t)
$$

to Equation (2.1) and get the equations for $r(t)$ and $\theta(t)$, 


$$
\left\{\begin{array}{l}
\theta^{\prime}=-\frac{1}{r} \phi(r \sin \theta) \sin \theta-\frac{1}{r} g(r \cos \theta) \cos \theta+\frac{1}{r} p(t) \cos \theta, \\
r^{\prime}=\phi(r \sin \theta) \cos \theta-g(r \cos \theta) \sin \theta+p(t) \sin \theta,
\end{array}\right.
$$

whenever $r(t) \neq 0$. Let $(r(t), \theta(t))=\left(r\left(t, r_{0}, \theta_{0}\right), \theta\left(t, r_{0}, \theta_{0}\right)\right)$ be the solution of Equation (2.2) through the initial point $(r(0), \theta(0))=\left(r_{0}, \theta_{0}\right)$.

Lemma 2.2. Assume that $\left(g_{1}\right)$ and $\left(g_{2}\right)$ hold. Then, for any fixed constant $T>0$, there exist positive constants $\alpha$ and $\gamma$ such that, for $r_{0} \geq \gamma$ and $t \in[0, T]$,

$$
\text { (1) } \alpha^{-1} r_{0} \leq r(t) \leq \alpha r_{0} ; \quad \text { (2) } \theta^{\prime}(t)<0 \text {. }
$$

Proof. (1) Since $\phi$ and $p$ are bounded, it follows from $\left(g_{2}\right)$ that there is a constant $c>0$ such that

$$
\left|r^{\prime}\right| \leq a r+c
$$

It follows that

$$
r_{0} \mathrm{e}^{-a t}-\frac{a}{c}\left(1-\mathrm{e}^{-a t}\right) \leq r(t) \leq r_{0} \mathrm{e}^{a t}+\frac{c}{a}\left(\mathrm{e}^{a t}-1\right), t \in[0, T] .
$$

Hence,

$$
r_{0} \mathrm{e}^{-a T}-\frac{a}{c}\left(1-\mathrm{e}^{-a T}\right) \leq r(t) \leq r_{0} \mathrm{e}^{a T}+\frac{c}{a}\left(\mathrm{e}^{a T}-1\right), t \in[0, T] .
$$

Obviously, we have that

$$
\mathrm{e}^{-a T} \leq \liminf _{r_{0} \rightarrow \infty} \frac{r(t)}{r_{0}} \leq \limsup _{r_{0} \rightarrow \infty} \frac{r(t)}{r_{0}} \leq \mathrm{e}^{a T}
$$

uniformly with respect to $t \in[0, T]$. Consequently, there are constants $\alpha>0$ and $\gamma_{0}>0$ such that, for $r_{0} \geq \gamma_{0}$ and $t \in[0, T]$,

$$
\alpha^{-1} r_{0} \leq r(t) \leq \alpha r_{0} \text {. }
$$

(2) From $\left(g_{1}\right)$ we know that there exist $d>0$ and $\delta>0$ such that

$$
\operatorname{sgn}(x) g(x)>\|p\|_{\infty},|x| \geq d
$$

and

$$
|g(x)-p(t)| \leq \delta,|x| \leq d, t \in \mathbf{R} .
$$

Therefore, if $|x(t)| \geq d$, then we infer from (2.3) that

$$
\frac{1}{r}(g(r \cos \theta)-p(t)) \cos \theta>0 .
$$

Since $\phi(y) y \geq 0$ for any $y \in \mathbf{R}$, we have that

$$
\frac{1}{r} \phi(r \sin \theta) \sin \theta \geq 0 \text {. }
$$

Consequently, if $|x(t)| \geq d$, then

$$
\theta^{\prime}(t)=-\frac{1}{r} \phi(r \sin \theta) \sin \theta-\frac{1}{r}(g(r \cos \theta)-p(t)) \cos \theta<0 .
$$

On the other hand, if $|x(t)| \leq d$, then we know from the conclusion in (1) 
that, for $r_{0}$ large enough, $|\cos \theta(t)| \leq \frac{d}{r(t)} \leq \frac{d \alpha}{r_{0}} \leq \frac{1}{8 \delta}$. It follows from (2.4) that

$$
\frac{1}{r}|(g(r \cos \theta)-p(t)) \cos \theta| \leq \frac{1}{8 r}
$$

provided that $r_{0}$ is large enough. From the expression of $\phi$ we know

$$
\lim _{|y| \rightarrow+\infty} \phi(y)=\lim _{|y| \rightarrow \infty} \frac{|y|}{\sqrt{1+y^{2}}}=1 .
$$

Furthermore, there exists $\zeta>0$ such that, for $|y| \geq \zeta$,

$$
|\phi(y)| \geq \frac{1}{2} .
$$

Therefore, if $r_{0}$ is large enough and $|x(t)| \leq d$, then we have

$$
|y(t)|=\sqrt{r^{2}(t)-x^{2}(t)} \geq \sqrt{\frac{r_{0}^{2}}{\alpha^{2}}-d^{2}} \geq \zeta
$$

and

$$
|\sin \theta(t)| \geq \frac{\sqrt{r^{2}(t)-d^{2}}}{r(t)} \geq \sqrt{1-\frac{d^{2}}{\alpha^{2} r_{0}^{2}}} \geq \frac{1}{2}
$$

which, together with (2.6), implies that

$$
\frac{1}{r} \phi(r \sin \theta) \sin \theta \geq \frac{1}{4 r} \text {. }
$$

It follows from (2.4) and (2.7) that, for $r_{0}$ large enough and $|x(t)| \leq d$,

$$
\begin{aligned}
\theta^{\prime}(t) & =-\frac{1}{r} \phi(r \sin \theta) \sin \theta-\frac{1}{r}(g(r \cos \theta)-p(t)) \cos \theta \\
& \leq-\frac{1}{4 r}+\frac{1}{8 r}=-\frac{1}{8 r}<0 .
\end{aligned}
$$

The proof of Lemma 2.2 is complete.

Remark 2.3. From the proof of Lemma 2.2 we know that there exists a constant $\gamma_{*}>0$ such that, if $r(t) \geq \gamma_{*}, t \in I$, then $\theta^{\prime}(t)<0, t \in I$, where $I$ is an interval.

Lemma 2.4. Assume that $\left(g_{1}\right)$ and $\left(g_{2}\right)$ hold. Then, for any $n \in \mathbf{N}$, there exists $R_{n}>0$ such that, for $r_{0} \geq R_{n}$,

$$
-\pi<\theta(2 n \pi)-\theta_{0}<0 .
$$

Proof. Since

$$
\lim _{|y| \rightarrow \infty} \frac{\phi(y)}{y}=\lim _{|y| \rightarrow \infty} \frac{1}{\sqrt{1+y^{2}}}=0,
$$

we have that, for any sufficiently small $\varepsilon>0$, there exists $c_{\varepsilon}>0$ such that

$$
0<\frac{\phi(y)}{y}<\varepsilon,|y| \geq c_{\varepsilon} .
$$


Set

$$
\begin{gathered}
D_{1}=\left\{(x, y) \in \mathbf{R}^{2}: x \geq 0, y \geq c_{\varepsilon}\right\}, D_{2}=\left\{(x, y) \in \mathbf{R}^{2}: x \leq 0, y \geq c_{\varepsilon}\right\}, \\
D_{3}=\left\{(x, y) \in \mathbf{R}^{2}: x \leq 0, y \leq-c_{\varepsilon}\right\}, D_{4}=\left\{(x, y) \in \mathbf{R}^{2}: x \geq 0, y \leq-c_{\varepsilon}\right\} .
\end{gathered}
$$

Next, we shall estimate the time needed for the solution $(x(t), y(t))$ to pass through each region of $D_{i} \quad(i=1,2,3,4)$, respectively. If $(x(t), y(t)) \in D_{1}$, $t \in\left[t_{1}, t_{2}\right]$ and $x\left(t_{1}\right)=0, y\left(t_{2}\right)=c_{\varepsilon}$, then we get from Lemma 2.2 and $\left(g_{2}\right)$ that, for $t \in\left[t_{1}, t_{2}\right]$ and $r_{0}$ large enough,

$$
\begin{aligned}
0>\theta^{\prime}(t) & \geq-\varepsilon \sin ^{2} \theta-a \cos ^{2} \theta-\frac{\alpha}{r_{0}}\left(b+\|p\|_{\infty}\right) \cos \theta \\
& \geq-\varepsilon \sin ^{2} \theta-a \cos ^{2} \theta-\varepsilon \\
& =-2 \varepsilon \sin ^{2} \theta-(a+\varepsilon) \cos ^{2} \theta .
\end{aligned}
$$

Consequently, we have

$$
t_{2}-t_{1} \geq \int_{\theta\left(t_{2}\right)}^{\theta\left(t_{1}\right)} \frac{\mathrm{d} \theta}{2 \varepsilon \sin ^{2} \theta+(a+\varepsilon) \cos ^{2} \theta} .
$$

Owing to $-\frac{\pi}{2}<\theta\left(t_{2}\right)-\theta\left(t_{1}\right)<0$ and $\theta\left(t_{1}\right)=\frac{\pi}{2}(\bmod \pi)$,

$\theta\left(t_{2}\right)=o(1)(\bmod \pi)$ for $r_{0} \rightarrow \infty$, we obtain

$$
t_{2}-t_{1} \geq \int_{o(1)}^{\frac{\pi}{2}} \frac{\mathrm{d} \theta}{2 \varepsilon \sin ^{2} \theta+(a+\varepsilon) \cos ^{2} \theta}=\sqrt{\frac{1}{2 \varepsilon(a+\varepsilon)}}\left(\frac{\pi}{2}+o(1)\right)>2 n \pi
$$

provided that $\varepsilon$ is small enough and $r_{0}$ is large enough. Similarly, we can prove that the time needed for the solution $(x(t), y(t))$ to pass through each region of $D_{i} \quad(i=2,3,4)$ is greater than $2 n \pi$ provided that $r_{0}$ is large enough. Therefore, The conclusion of Lemma 2.4 holds.

Lemma 2.5. Assume that $\left(g_{1}\right)$ and $\left(g_{2}\right)$ hold. Then for any $n \in \mathbf{N}$ and $t_{0} \in \mathbf{R}$, there exists an $t_{*}>t_{0}$ such that

$$
\theta\left(t_{*}\right)-\theta\left(t_{0}\right)<-2 n \pi
$$

provided that $r\left(t_{0}\right)$ is large enough.

Proof. Assume by contradicition that there is an integer $l>0$ such that

$$
\theta(t)-\theta\left(t_{0}\right)>-2 l \pi
$$

for any sufficiently large $r\left(t_{0}\right)>0$ and $t>t_{0}$. We will proceed in two cases.

(1) For $t \in\left[t_{0},+\infty\right), r(t) \geq \gamma_{*}$, where $\gamma_{*}$ is defined in Remark 2.3. In this case, $\theta^{\prime}(t)<0$ and then $\theta(t)$ is decreasing on the interval $\left[t_{0},+\infty\right)$. From (2.9) we know that

$$
\lim _{t \rightarrow+\infty} \theta(t)=\vartheta>-\infty .
$$

Therefore, the orbit $(x(t), y(t))$ has a asymptotical ray $\theta=\vartheta$. If $\vartheta=\frac{\pi}{2}(\bmod \pi)$, then $x(t) \rightarrow 0$ as $t \rightarrow+\infty$. It follows that, for $t$ large enough, $|y(t)| \geq 1$ and then 


$$
\left|x^{\prime}(t)\right|=|\phi(y(t))|=\frac{|y(t)|}{\sqrt{1+y^{2}(t)}} \geq \frac{1}{\sqrt{2}},
$$

which implies that $x(t) \rightarrow \pm \infty$ as $t \rightarrow+\infty$. This is a contradicition. Hence, $x(t) \rightarrow \infty, t \rightarrow+\infty$. Without loss of generality, we assume the asymptotical ray is $y=k x, \quad x \geq 0$, where $k=\tan \vartheta$. If $k \geq 0$, then we have $x(t) \rightarrow+\infty, y(t) \rightarrow+\infty$ as $t \rightarrow+\infty$. But, it follows from $y^{\prime}(t)=-g(x(t))+p(t)$ that there is a sufficiently large constant $\beta>0$ such that $y^{\prime}(t) \leq-\beta$ for $t>0$ large enough, which implies $y(t) \rightarrow-\infty$ as $t \rightarrow+\infty$. This is a contradicition. If $k<0$, then $x(t) \rightarrow+\infty, y(t) \rightarrow-\infty$ as $t \rightarrow+\infty$. But, since $x^{\prime}(t)=\phi(y(t))=\frac{y(t)}{\sqrt{1+y^{2}(t)}}$, we have that $x^{\prime}(t)<0$ for $t$ large enough. Consequently, $x(t)$ is bounded from above. Thus we get a contradicition.

(2) There is an $t_{*}>t_{0}$ such that $r\left(t_{*}\right)=\gamma_{*}$ and $r(t)>\gamma_{*}, t \in\left[t_{0}, t_{*}\right)$. We next show that there is a large $R_{0}>\gamma_{*}$ such that, for $r\left(t_{0}\right) \geq R_{0}$,

$$
\theta\left(t_{*}\right)-\theta\left(t_{0}\right)<-2 l \pi \text {. }
$$

To this end, we shall construct a continuous counter-clockwise rotating spiral curve $\sigma:[0,+\infty) \rightarrow \mathbf{R}^{2}$, which is injective and makes infinite rotations around the origin $(0,0)$. Moreover, the curve $\sigma$ satisfies

$$
\lim _{s \rightarrow+\infty}|\sigma(s)|=+\infty
$$

and every time when the solution $(x(t), y(t))$ of Equation (2.1) intersects with the curve $\sigma$ only from the inner part to the outer part. Let us take a positive constant $M>0$ with $\|p\|_{\infty}<M$. We define

$$
W_{+}(x, y)=\Phi(y)+G(x)+M x, \quad y \geq 0
$$

and

$$
W_{-}(x, y)=\Phi(y)+G(x)-M x, \quad y \leq 0 .
$$

Set

$$
W_{+}(t)=\Phi(y(t))+G(x(t))+M x(t), y(t) \geq 0,
$$

and

$$
W_{-}(t)=\Phi(y(t))+G(x(t))-M x(t), \quad y(t) \leq 0 .
$$

Then we have

$$
W_{+}^{\prime}(t)=(p(t)+M) \phi(y(t))>0, \text { for } y(t)>0
$$

and

$$
W_{-}^{\prime}(t)=(p(t)-M) \phi(y(t))>0 \text {, for } y(t)<0 .
$$

We now take a large constant $c>\gamma_{*}$ such that the curve $\Phi(y)+G(x)+M x=c$ is a simply closed curve and the circle $x^{2}+y^{2}=\gamma_{*}^{2}$ lies inside this closed curve. Consider the curve 


$$
\Gamma_{1}^{+}: \Phi(y)+G(x)+M x=c, \quad y \geq 0,
$$

which intersects with the $x$-axis at exactly two points $\left(x_{+}(c), 0\right)$ and $\left(x_{-}(c), 0\right)$ with $x_{-}(c)<0<x_{+}(c)$. Then we have

$$
G\left(x_{+}(c)\right)+M x_{+}(c)=c, G\left(x_{-}(c)\right)+M x_{-}(c)=c .
$$

Set

$$
c_{1}=G\left(x_{-}(c)\right)-M x_{-}(c) .
$$

Let us consider the curve

$$
\Gamma_{1}^{-}: \Phi(y)+G(x)-M x=c_{1}, \quad y \leq 0 .
$$

Assume that $\Gamma_{1}^{-}$intersects with the positive $x$-axis at the point $\left(x_{+}\left(c_{1}\right), 0\right)$. Then we have

$$
G\left(x_{+}\left(c_{1}\right)\right)-M x_{+}\left(c_{1}\right)=c_{1} .
$$

We shall prove that $x_{+}\left(c_{1}\right)>x_{+}(c)$. In fact, since

$$
\begin{aligned}
G\left(x_{+}\left(c_{1}\right)\right)-M x_{+}\left(c_{1}\right) & =c_{1}=G\left(x_{-}(c)\right)-M x_{-}(c) \\
& =c-2 M x_{-}(c)>G\left(x_{+}(c)\right)+M x_{+}(c),
\end{aligned}
$$

we get

$$
G\left(x_{+}\left(c_{1}\right)\right)>G\left(x_{+}(c)\right),
$$

which implies that $x_{+}\left(c_{1}\right)>x_{+}(c)$ for $c$ large enough because $G(x)$ is increasing for $x>0$ large enough. Set

$$
c_{2}=G\left(x_{+}\left(c_{1}\right)\right)+M x_{+}\left(c_{1}\right) .
$$

Next we consider the curve

$$
\Gamma_{2}^{+}: \Phi(y)+G(x)+M x=c_{2}, \quad y \geq 0 .
$$

Applying the same method as above we can define the curve $\Gamma_{2}^{-}$. Successively, we can construct the curves $\Gamma_{i}^{+}$and $\Gamma_{i}^{-}(i=3,4, \cdots)$. Let us set

$$
\Gamma=\bigcup_{i=1}^{\infty}\left(\Gamma_{i}^{+} \cup \Gamma_{i}^{-}\right) \text {. }
$$

We now take a starting point $\left(x_{+}(c), 0\right)$ and define the parametrization of $\Gamma$ in polar coordinates

$$
\sigma(s)=(\varsigma(s) \cos s, \varsigma(s) \sin s), s \in[0,+\infty),
$$

where $s(s)$ denotes the Euclidian norm of a point on $\Gamma$, whose argument is $s$. From the construction of $\Gamma$ we know that its parametrization $\sigma(s)$ is continuous and satisfies (2.10) and $\sigma(s)$ makes infinite rotations around the origin $(0,0)$ as $s \rightarrow+\infty$. Moreover, it follows from (2.11) and (2.12) that all solutions cross the curve only from the inner part to the outer part.

For the fixed integer $l>0$ above. Let us take a sufficiently large constant $R_{0}>0$ such that the spiral curve $\sigma(s)(s \in[0,2(l+1) \pi])$ lies inside the circle $x^{2}+y^{2}=R_{0}^{2}$. If $r\left(t_{0}\right) \geq R_{0}$ and there is a sufficiently large $t_{*}>0$ such 
that

$$
r\left(t_{*}\right)=\gamma_{*}, r(t)>\gamma_{*}, t \in\left[t_{0}, t_{*}\right),
$$

then the orbit $(r(t), \theta(t))$ will move clock-wise during the period $\left[t_{0}, t_{*}\right]$. Since $(r(t), \theta(t))$ can cross spiral $\sigma(s)$ only from the inner part to the outer part, it will make at least $l$ rotations when it finally reaches the circle $x^{2}+y^{2}=\gamma_{*}^{2}$. Consequently, we get

$$
\theta\left(t_{*}\right)-\theta\left(t_{0}\right)<-2 l \pi
$$

which contradicts with (2.9)

\section{Estimates on the Angle Variable}

When the condition $\left(g_{1}\right)$ holds, it was proved in [4] that Equation (2.1) has at least one $2 \pi$-periodic solution.

Let $\left(x_{0}(t), y_{0}(t)\right)$ be an $2 \pi$-periodic solution of Equation (2.1). We now take a transformation

$$
x=u+x_{0}(t), \quad y=v+y_{0}(t)
$$

to Equation (2.1) and get the equations for $u(t)$ and $v(t)$,

$$
\left\{\begin{array}{l}
\frac{\mathrm{d} u}{\mathrm{~d} t}=\phi\left(v+y_{0}(t)\right)-\phi\left(y_{0}(t)\right), \\
\frac{\mathrm{d} v}{\mathrm{~d} t}=-g\left(u+x_{0}(t)\right)+g\left(x_{0}(t)\right) .
\end{array}\right.
$$

Let $(u(t), v(t))=\left(u\left(t, u_{0}, v_{0}\right), v\left(t, u_{0}, v_{0}\right)\right)$ be the solution of Equation (3.1) satisfying the initial value $(u(0), v(0))=\left(u_{0}, v_{0}\right)$. From Lemma 2.1 we know that $(u(t), v(t))$ exists on the whole $t$-axis uniquely. Thus we can define the Poincaré map $P$ of Equation (3.1),

$$
P:\left(u_{0}, v_{0}\right) \rightarrow(u(2 \pi), v(2 \pi)) .
$$

It is well-known that $P$ is an area-preserving homeomorphism.

Obviously, Equation (3.1) has a trivial solution $(u(t), v(t))=(0,0) \quad(t \in \mathbf{R})$, which corresponds to the $2 \pi$-periodic solution $\left(x_{0}(t), y_{0}(t)\right)$. Let $(u(t), v(t))$ be the solution of Equation (3.1) satisfying the initial condition $\left(u_{0}, v_{0}\right) \neq(0,0)$. It follows that $(u(t), v(t)) \neq(0,0)$ for all $t \in R$. Hence, it can be represented by polar coordinates

$$
u(t)=\rho(t) \cos \psi(t), v(t)=\rho(t) \sin \psi(t) .
$$

where $\rho(t)>0$ and $\psi(t)$ are continuous for all $t \in \mathbf{R}$.

Using a similar method as in proving Lemma 2.2, we can prove the following lemma.

Lemma 3.1. Assume that $\left(g_{1}\right)$ and $\left(g_{2}\right)$ hold. Then there is an $\rho_{0}>0$ such that, if $\rho(t) \geq \rho_{0}, t \in I$, then

$$
\psi^{\prime}(t)<0, t \in I,
$$

where $I$ is an interval. 
Lemma 3.2. If $t_{1}$ and $m$ are two positive constants such that

$$
\psi\left(t_{1}\right)-\psi(0)<-2 m \pi
$$

then, for any $t_{2}>t_{1}$,

$$
\psi\left(t_{2}\right)-\psi\left(t_{1}\right)<-2 m \pi+\pi
$$

Proof. The proof follows an argument in [13]. Since $\phi^{\prime}(y)=\frac{1}{\sqrt{\left(1+y^{2}\right)^{3}}}$ and $g$ is locally Lipschitz continuous, the solutions of Cauchy problems of Equation (3.1) are unique. Therefore, the solution $(u(t), v(t))$ can not go through the origin. Obviously, $\phi(y)$ is increasing. Since $u^{\prime}(t)=\phi\left(v(t)+y_{0}(t)\right)-\phi\left(y_{0}(t)\right)$, the orbit $(u(t), v(t))$ moves in the clockwise direction when it intersects with the $v$-axis. Therefore, if the orbit $(x(t), y(t))$ intersects the positive (or the negative) $v$-axis at the time $t=\alpha>0$ and intersects subsequently the negative (or the positive) $v$-axis at the time $t=\beta>\alpha$, then we have

$$
\psi(\beta)-\psi(\alpha)=-\pi \text {. }
$$

On the other hand, if the orbit $(x(t), y(t))$ stays in the right half-plane (or in the left half-plane) during the time interval $\alpha \leq t \leq \beta$, the increase of the angle satisfies

$$
\psi(\beta)-\psi(\alpha)<\pi
$$

It follows from (3.2) and (3.3) that

$$
\psi\left(t_{2}\right)-\psi(0)=\left[\psi\left(t_{1}\right)-\psi(0)\right]+\left[\psi\left(t_{2}\right)-\psi\left(t_{1}\right)\right]<-2 m \pi+\pi .
$$

Lemma 3.3. Assume that $\left(g_{1}\right)$ and $\left(g_{2}\right)$ hold. Then, for any $n \in \mathbf{N}$, there exists $\rho_{n}>0$ such that, for $\rho(0) \geq \rho_{n}$,

$$
-2 \pi<\psi(2 n \pi)-\psi(0)<0 .
$$

Proof. We denote by $L_{1}$ the orbit of the $2 \pi$-periodic solution $\left(x_{0}(t), y_{0}(t)\right)$ of Equation (2.1) in the $(x, y)$-plane. Let $L_{2}$ be the orbit of the solution $(x(t), y(t))$ of Equation (2.1) satisfying the initial value $(x(0), y(0)) \in \mathbf{R}^{2}$ in the $(x, y)$-plane. Consider the moving points

$$
A=\left(x_{0}(t), y_{0}(t)\right) \in L_{1}, B=(x(t), y(t)) \in L_{2} .
$$

Let $\Delta_{O A B}$ be the triangle with the vertices $O, A, B$. Obviously, the vector $\boldsymbol{O B}$ has the argument $\theta(t)$ and the vector $\boldsymbol{A B}$ has the argument $\psi(t)$. It follows from Lemma 2.2 that, if $x(0)^{2}+y(0)^{2}$ is large enough, then $x(t)^{2}+y(t)^{2}$ is also large enough for $t \in[0,2 n \pi]$ and then we have $|\angle O B A|<\frac{\pi}{6}$. Furthermore,

$$
\theta(t)=\psi(t)+\angle O B A, t \in[0,2 n \pi] .
$$

Therefore, we have

$$
|\theta(2 n \pi)-\psi(2 n \pi)| \leq \frac{\pi}{6},|\theta(0)-\psi(0)| \leq \frac{\pi}{6} .
$$


From Lemma 2.4, Lemma 3.1 and (3.4) we get

$$
\begin{aligned}
0 & >\psi(2 n \pi)-\psi(0) \\
& \geq(\theta(2 n \pi)-\theta(0))-|\psi(2 n \pi)-\theta(2 n \pi)|-|\psi(0)-\theta(0)| \\
& \geq-\pi-\frac{\pi}{3}>-2 \pi .
\end{aligned}
$$

Lemma 3.4. Assume that $\left(g_{1}\right),\left(g_{2}\right)$ hold. Then for any $n \in \mathbf{N}$ and $t_{0} \in \mathbf{R}$, there is an $\rho_{n}^{\prime}>0$ such that for $\rho\left(t_{0}\right) \geq \rho_{n}^{\prime}$ and any sufficiently large $t>t_{0}$,

$$
\psi(t)-\psi\left(t_{0}\right)<-2 n \pi+\frac{4 \pi}{3} .
$$

Proof. We still use some notations in the proof of Lemma 3.3. From the proof of Lemma 2.5 we know that we can enlarge $\gamma_{*}>0$ such that $|\angle O B A|<\frac{\pi}{6}$ and

$$
\theta(t)=\psi(t)+\angle O B A, t \in\left[t_{0}, t_{*}\right]
$$

where $t_{*}$ is a constant given in Lemma 2.5. Then we have

$$
\left|\theta\left(t_{*}\right)-\psi\left(t_{*}\right)\right| \leq \frac{\pi}{6},\left|\theta\left(t_{0}\right)-\psi\left(t_{0}\right)\right| \leq \frac{\pi}{6} .
$$

From Lemma 2.5 and (3.5) we get that, for $\rho\left(t_{0}\right)$ (or $r\left(t_{0}\right)$ ) large enough,

$$
\begin{aligned}
& \psi\left(t_{*}\right)-\psi\left(t_{0}\right) \\
& \leq \theta\left(t_{*}\right)-\theta\left(t_{0}\right)+\left|\psi\left(t_{*}\right)-\theta\left(t_{*}\right)\right|+\left|\psi\left(t_{0}\right)-\theta\left(t_{0}\right)\right| \\
& <-2 n \pi+\frac{\pi}{3} .
\end{aligned}
$$

According to Lemma 3.2, we get that, for any $t>t_{*}$,

$$
\psi(t)-\psi\left(t_{0}\right)<-2 n \pi+\frac{4 \pi}{3} .
$$

\section{Proof of Main Theorem}

We first recall a generalized version of the Poincaré-Birkhoff fixed point theorem by Rebelo [12].

A generalized form of the Poincaré-Birkhoff fixed point theorem Let $\mathcal{A}$ be an annular region bounded by two strictly star-shaped curves around the origin, $\Gamma_{1}$ and $\Gamma_{2}, \Gamma_{1} \subset \operatorname{int}\left(\Gamma_{2}\right)$, where int $\left(\Gamma_{2}\right)$ denotes the interior domain bounded by $\Gamma_{2}$. Suppose that $F: \overline{\operatorname{int}\left(\Gamma_{2}\right)} \rightarrow R^{2}$ is an area-preserving homeomorphism and $F \mid \mathcal{A}$ admits a lifting, with the standard covering projection $\Pi:(r, \theta) \rightarrow z=(r \cos \theta, r \sin \theta)$, of the form

$$
\tilde{F} \mid \mathcal{A}:(r, \theta) \rightarrow(w(r, \theta), \theta+h(r, \theta)),
$$

where $w$ and $h$ are continuous functions of period $2 \pi$ in the second variable. Correspondingly, for $\tilde{\Gamma}_{1}=\Pi^{-1}\left(\Gamma_{1}\right)$ and $\tilde{\Gamma}_{2}=\Pi^{-1}\left(\Gamma_{2}\right)$, assume the twist condition

$$
h(r, \theta)>0 \text { on } \tilde{\Gamma}_{1} ; \quad h(r, \theta)<0 \text { on } \tilde{\Gamma}_{2}
$$


or

$$
h(r, \theta)<0 \text { on } \tilde{\Gamma}_{1} ; \quad h(r, \theta)>0 \text { on } \tilde{\Gamma}_{2} .
$$

Then, $F$ has two fixed points $z_{1}, \quad z_{2}$ in the interior of $\mathcal{A}$, such that

$$
h\left(\Pi^{-1}\left(z_{1}\right)\right)=h\left(\Pi^{-1}\left(z_{2}\right)\right)=0 .
$$

Proof of Theorem 1.1. According to Lemma 3.4, we can take a prime $q \geq 2$ and a sufficiently large constant $\varrho_{*}>\varrho_{0} \quad\left(\varrho_{0}\right.$ is defined in Lemma 3.1) such that, every solution $(u(t), v(t))$ of Equation (3.1) with $u\left(t_{0}\right)^{2}+v\left(t_{0}\right)^{2}=\varrho_{*}^{2}$ and $t_{0} \in[0,2 \pi]$, satisfies the following property:

$(P)$ There is a constant $t_{*}>0$ such that

$$
\psi\left(t_{*}+t_{0}\right)-\psi\left(t_{0}\right)<-(2 q+3) \pi+\frac{4 \pi}{3} .
$$

Set

$$
S=\left\{t_{*}>0: \text { the property }(\mathrm{P}) \text { holds }\right\} \text {. }
$$

Since $\left\{(u, v): u^{2}+v^{2}=\varrho_{*}^{2}\right\}$ is compact and the solution $(u(t), v(t))$ is continuous dependence on the initial value $\left(t_{0}, u_{0}, v_{0}\right)$, we can take a suitable $t_{*}$ for every solution $(u(t), v(t))$ such that $S$ is bounded from above. Write

$$
T_{*}=\sup S \text {. }
$$

Choosing $n_{0}=\max \left\{2,\left[T_{*} / 2 \pi\right]+1\right\}$, we infer from Lemma 3.2 that, for any $n \geq n_{0}$

$$
\psi(2 n \pi)-\psi(0)<-2 q \pi, \rho(0)=\varrho_{*} .
$$

It follows from Lemma 3.3 that there is a sufficiently large constant $a_{n}>\varrho_{*}$ such that

$$
\psi(2 n \pi)-\psi(0)>-2 \pi, \rho(0)=a_{n}
$$

From (4.2) and (4.3) we know that the $n$-iteration $P^{n}$ of the Poincaré map $P$ is twisting on the annulus:

$$
D_{n}: \varrho_{*}^{2} \leq u^{2}+v^{2} \leq a_{n}^{2} .
$$

Obviously, $P^{n}$ is an area-preserving homeomorphism. According to the generalized Poincaré-Birkhoff fixed point theorem, $P^{n}$ has at least two fixed points $\left(u_{n}^{i}, v_{n}^{i}\right) \in D_{n}(i=1,2)$, whose polar coordinates are $\left(\rho_{n}^{i}, \psi_{n}^{i}\right)$, satisfying

$$
\psi(2 n \pi)-\psi(0)=-2 q \pi
$$

with $(\rho(0), \psi(0))=\left(\rho_{n}^{i}, \psi_{n}^{i}\right), \quad(i=1,2)$. It follows that

$$
\left(u_{n, i}(t), v_{n, i}(t)\right)=\left(u\left(t, u_{n}^{i}, v_{n}^{i}\right), v\left(t, u_{n}^{i}, v_{n}^{i}\right)\right)
$$

are the $2 n \pi$-periodic solutions of (3.1). Using standard methods as in [14] and (4.4), we can further prove that $2 n \pi$ is the minimal period. Therefore, $\left(x_{n, i}(t), y_{n, i}(t)\right)=\left(u_{n, i}(t)+x_{0}(t), v_{n, i}(t)+y_{0}(t)\right)$ are subharmonic solutions of order $n$ of Equation (2.1). 
In what follows, we shall prove

$$
\lim _{n \rightarrow \infty}\left(\min _{t \in[0,2 n \pi]}\left(u_{n, i}^{2}(t)+v_{n, i}^{2}(t)\right)\right)=+\infty,(i=1,2) .
$$

Firstly, we prove

$$
u_{n, i}^{2}(t)+v_{n, i}^{2}(t)>\rho_{*}^{2}, t \in[0,2 n \pi] .
$$

Otherwise, there are $t_{*}^{i} \in[0,2 n \pi] \quad(i=1,2)$ such that

$$
u_{n, i}^{2}\left(t_{*}^{i}\right)+v_{n, i}^{2}\left(t_{*}^{i}\right)=\rho_{*}^{2} .
$$

Write $t_{*}^{i}=2 k_{i} \pi+\tau_{*}^{i}, \quad 0 \leq k_{i} \leq n, \quad 0 \leq \tau_{*}^{i}<2 \pi$. Set

$$
\bar{u}_{n, i}(t)=u_{n, i}\left(t+2 k_{i} \pi\right), \bar{v}_{n, i}(t)=v_{n, i}\left(t+2 k_{i} \pi\right) .
$$

Obviously, $\left(\bar{u}_{n, i}(t), \bar{v}_{n, i}(t)\right)$ are the $2 n \pi$-periodic solutions of Equation (3.1) satisfying

$$
\bar{u}_{n, i}^{2}\left(\tau_{*}^{i}\right)+\bar{v}_{n, i}^{2}\left(\tau_{*}^{i}\right)=\rho_{*}^{2}
$$

Let $\left(\bar{\rho}_{n, i}(t), \bar{\psi}_{n, i}(t)\right)$ be the polar coordinates expression of $\left(\bar{u}_{n, i}(t), \bar{v}_{n, i}(t)\right)$. From the definition of $n_{0}$ and (4.1), (4.7) we know that, for $n \geq n_{0}$,

$$
\bar{\psi}_{n, i}\left(2 n \pi+\tau_{*}^{i}\right)-\bar{\psi}_{n, i}\left(\tau_{*}^{i}\right)<-2 q \pi,
$$

which contradicits with (4.4) because the orbits of the solutions $\left(u_{n, i}(t), v_{n, i}(t)\right)$ and $\left(\bar{u}_{n, i}(t), \bar{v}_{n, i}(t)\right)$ are the same.

Secondly, we prove

$$
\lim _{n \rightarrow \infty}\left(\max _{t \in[0,2 n \pi]}\left(u_{n, i}^{2}(t)+v_{n, i}^{2}(t)\right)\right)=+\infty,(i=1,2) .
$$

Otherwise, there are a subsequence $\left(u_{n_{k}, i}(t), v_{n_{k}, i}(t)\right)$ and a constant $b>0$ such that, for $n_{k} \geq n_{0}$,

$$
\rho_{*}^{2} \leq u_{n_{k}, i}^{2}(t)+v_{n_{k}, i}^{2}(t) \leq b^{2}, t \in\left[0,2 n_{k} \pi\right] .
$$

Set

$$
D=\left\{(u, v) \in \mathbf{R}^{2}: \rho_{*}^{2} \leq u^{2}+v^{2} \leq b^{2}\right\} .
$$

Since $D$ is compact, it follows from Lemma 3.1 that there is a constant $c_{0}>0$ such that, if a solution $(u(t), v(t))(t \in I, I$ is an interval) of Equation (3.1) lies in $D$, then

$$
\psi^{\prime}(t) \leq-c_{0}, t \in I
$$

Let us denote by $\left(\rho_{n, i}(t), \psi_{n, i}(t)\right)$ the polar coordinates of $\left(u_{n, i}(t), v_{n, i}(t)\right)$. Then we get from (4.4) and (4.9) that

$$
-2 q \pi=\psi_{n_{k}, i}\left(2 n_{k} \pi\right)-\psi_{n_{k}, i}(0) \leq-2 c_{0} n_{k} \pi,
$$

which implies

$$
q \geq c_{0} n_{k} .
$$

This is impossible since $n_{k} \rightarrow+\infty$ as $k \rightarrow \infty$.

Finally, we prove (4.5). Assume by contradicition that (4.5) does not hold. Then we know from (4.8) that there exist a subsequence $\left(u_{n_{l}, i}(t), v_{n_{l}, i}(t)\right)$ and 
a constant $d_{0}>0$ such that

$$
\rho_{*}^{2} \leq \min _{t \in\left[0,2 n_{l} \pi\right]}\left(u_{n_{l}, i}^{2}(t)+v_{n_{l}, i}^{2}(t)\right) \leq d_{0}^{2} \leq \max _{t \in\left[0,2 n_{l} \pi\right]}\left(u_{n_{l}, i}^{2}(t)+v_{n_{l}, i}^{2}(t)\right) \rightarrow+\infty,(l \rightarrow \infty) .
$$

Since $\left(u_{n_{l}, i}(t), v_{n_{l}, i}(t)\right)$ are $2 n_{l} \pi$-periodic, there are $t_{n_{l}}^{i} \in\left[0,2 n_{l} \pi\right]$ and $\bar{t}_{n_{l}}^{i} \in\left[t_{n_{l}}^{i}, t_{n_{l}}^{i}+2 n_{l} \pi\right](i=1,2)$ such that

$$
\max _{t \in\left[0,2 n_{l} \pi\right]}\left(u_{n_{l}, i}^{2}(t)+v_{n_{l}, i}^{2}(t)\right)=u_{n_{l}, i}^{2}\left(t_{n_{l}}^{i}\right)+v_{n_{l}, i}^{2}\left(t_{n_{l}}^{i}\right) \rightarrow+\infty,(l \rightarrow \infty)
$$

and

$$
\min _{t \in\left[0,2 n_{l} \pi\right]}\left(u_{n_{l}, i}^{2}(t)+v_{n_{l}, i}^{2}(t)\right)=u_{n_{l}, i}^{2}\left(\bar{t}_{n_{l}}^{i}\right)+v_{n_{l}, i}^{2}\left(\bar{t}_{n_{l}}^{i}\right) \leq d_{0}^{2}
$$

Using the similar method as in proving Lemma 3.4, we can prove that, for 1 large enough,

$$
\psi_{n_{l}, i}\left(\bar{t}_{n_{l}}^{i}\right)-\psi_{n_{l}, i}\left(t_{n_{l}}^{i}\right)<-2 q \pi
$$

From Lemma 3.1 we know that, for Ilarge enough,

$$
\psi_{n_{l}, i}\left(t_{n_{l}}^{i}+2 n_{l} \pi\right)-\psi_{n_{l}, i}\left(t_{n_{l}}^{i}\right)<-2 q \pi
$$

which contradicts with (4.4).

According to (4.5), we know that, for any integer $n \geq n_{0}$, Equation (2.1) has at least two subharmonic solutions $\left(x_{n, i}(t), y_{n, i}(t)\right) \quad(i=1,2)$ of order $n$ satisfying

$$
\lim _{n \rightarrow \infty}\left(\min _{t \in[0,2 n \pi]}\left(x_{n, i}^{2}(t)+y_{n, i}^{2}(t)\right)\right)=+\infty,(i=1,2) \text {. }
$$

Since

$$
x_{n, i}^{\prime}(t)=\phi\left(y_{n, i}(t)\right)
$$

where $\phi$ is defined in section 2 , we have

$$
y_{n, i}(t)=\frac{x_{n, i}^{\prime}(t)}{\sqrt{1-x_{n, i}^{\prime 2}(t)}} .
$$

Consequently, we get from (4.10) that

$$
\lim _{n \rightarrow \infty}\left(\min _{t \in\left[0,2 n_{l} \pi\right]}\left(x_{n, i}^{2}(t)+\frac{x_{n, i}^{\prime}(t)^{2}}{1-x_{n, i}^{\prime 2}(t)}\right)\right)=+\infty .
$$

Furthermore,

$$
\lim _{n \rightarrow \infty}\left(\min _{t \in\left[0,2 n_{l} \pi\right]}\left(x_{n, i}^{2}(t)+\frac{1}{1-x_{n, i}^{\prime 2}(t)}\right)\right)=+\infty, \quad(i=1,2) .
$$

The proof is complete.

\section{Conflicts of Interest}

The authors declare no conflicts of interest regarding the publication of this paper. 


\section{References}

[1] Brezis, H. and Mawhin, J. (2010) Periodic Solutions of the Forced Relativistic Pendulum. Differential Integral Equations, 23, 801-810.

[2] Bereanu, C. and Torres, P.J. (2012) Existence of at Least Two Periodic Solutions of the Forced Relativistic Pendulum. Proceedings of the American Mathematical Society, 140, 2713-2719. https://doi.org/10.1090/S0002-9939-2011-11101-8

[3] Bereanu, C., Jebelean, P. and Mawhin, J. (2010) Periodic Solutions of Pendulum-Like Perturbations of Singular and Bounded $\phi$-Laplacians. Journal of Dynamics and Differential Equations, 22, 463-471. https://doi.org/10.1007/s10884-010-9172-3

[4] Bereanu, C. and Mawhin, J. (2007) Existence and Multiplicity Results for Some Nonlinear Problems with Singular $\phi$-Laplacian, Journal of Differential Equations, 243, 536-557. https://doi.org/10.1016/j.jde.2007.05.014

[5] Chu, J., Liang, Z., Liao, F. and Lu, S. (2017) Existence and Stability of Periodic Solutions for Relativistic Singular Equations. Communications on Pure and Applied Analysis, 16, 591-609. https://doi.org/10.3934/cpaa.2017029

[6] Torres, P.J. (2008) Periodic Oscillations of the Relativistic Pendulum with Friction. Physics Letters A, 372, 6386-6387. https://doi.org/10.1016/j.physleta.2008.08.060

[7] Han, Y. and Li, X. (2017) On Littlewoods Boundedness Problem for Relativistic Oscillators with Singular Potentials. Journal of Mathematical Analysis and Applications, 449, 1424-1471. https://doi.org/10.1016/j.jmaa.2016.12.081

[8] Liu, Q., Wang, C. and Wang, Z. (2014) On Littlewoods Boundedness Problem for Relativistic Oscillators with Anharmonic Potentials. Journal of Differential Equations, 257, 4542-4571. https://doi.org/10.1016/j.jde.2014.08.017

[9] Mawhin, J. and Villari, G. (2017) Periodic Solutions of Some Autonomous Liénard Equations with Relativistic Acceleration. Nonlinear Analysis, 160, 16-24. https://doi.org/10.1016/j.na.2017.05.001

[10] Marò, S. (2015) Relativistic Pendulum and Invariant Curves. Discrete \& Continuous Dynamical Systems, 35, 1139-1162. https://doi.org/10.3934/dcds.2015.35.1139

[11] Marò, S. (2013) Periodic Solutions of a Forced Relativistic Pendulum via Twist Dynamics. Topological Methods in Nonlinear Analysis, 42, 51-75.

[12] Rebelo, C. (1997) A Note on the Poincaré-Birkhoff Fixed Point Theorem and Periodic Solutions of Planar Systems. Nonlinear Analysis, 29, 291-311. https://doi.org/10.1016/S0362-546X(96)00065-X

[13] Ding, T. (2007) Approaches to the Qualitative Theory of Ordinary Differential Equations: Dynamical Systems and Nonlinear Oscillations. World Scientific Publishing, Singapore.

[14] Ding, T. and Zanolin, F. (1992) Periodic Solutions of Duffing's Equations with Super-Quadratic Potential. Journal of Differential Equations, 97, 328-378.

https://doi.org/10.1016/0022-0396(92)90076-Y 